\title{
Moisture study on nano-coated hearing aids
}

\begin{abstract}
The corrosive interaction of salt and metal are well known and highly documented ${ }^{1}$. However, there is a paucity of available research into the effects of perspiration, saltwater, or chlorinated water on hearing aids. "Nano coating" was developed to reduce the ability of moisture to enter the hearing aid shell. Nano-coating technology is being implemented by major hearing aid companies.

The purpose of this study was to examine the impact of moist environments on hearing aids in which nano coating technologies have been applied and to analyze and compare quantitative and qualitative data. The study included five hearing aid manufacturers, all of whom supplied hearing aids which were exposed to saltwater and chlorinated water for three exposure durations: 30 minutes, 180 minutes and 480 minutes.
\end{abstract}

Volume 4 Issue 4 - 2016

Danielle Hummel

Department of Speech and Hearing Sciences, Lamar University, USA

Correspondence: Danielle Hummel, AUD, Department of Speech and Hearing Sciences, Lamar University, Beaumont, Texas, USA, Email dhummel2186@gmail.com

Received: June 25, 2016 | Published: July 21, 2016
Abbreviations: BHI, better hearing institute; BTE, behindthe-ear; ITE, in-the-ear; VA, veterans administration; $\mathrm{NaCl}$, sodium chloride; WNL, within normal limits; EAA, electro acoustic analysis; THD, total harmonic distortion

\section{Introduction}

According to the Better Hearing Institute (BHI) Behind-the-Ear (BTE) hearing aids often demonstrate greater problems with moisture than in-the-ear (ITE) hearing aids. ${ }^{2}$ BHI noted moisture is difficult to observe and not always easy to diagnose as the cause of hearing aid failure. As public awareness of hearing loss increases, research evaluating moisture and corrosion should additionally be more widely available. Strom ${ }^{3}$ reported the private sector and the Veterans Administration (VA) continue to rely heavily on behind-the-ear (BTE) hearing aid technology (2011). The concept of Nano-coating technology is extensively accepted, and implemented by most major hearing aid manufacturers. Nano-coating was developed to help reduce moisture from entering hearing aids.

The study incorporated 30 hearing aids from five hearing aid manufacturers. Hearing aids were evaluated after being "misted" in saltwater and chlorinated water for three durations (30minutes, 180 minutes and 480 minutes) over 10 consecutive days.

At the conclusion of the study the investigator hoped to answer the following major questions:

a. Was moisture difficult to observe without electro acoustical analysis?

b. Were significant differences present in the relationship of exposure duration?

c. Were significant differences present between saltwater and chlorinated water?

d. Were significant differences present in the relationship of hearing aid function on days 1-5 and 6-10?

\section{Nano-coating technology}

Nano-coating technology does not impact or affect the electronic components of the hearing aid. Nano coating maintains the look and feel of the original components and product. Nano-coating technology can be used to resist water and oil (hydrophobic and oleophobic, respectively).

Nano-coating technology varies across manufacturers. For example, P2i markets itself as "The world leader in liquid repellent Nano-coating technology,". ${ }^{4}$ P2i Ltd was established in 2004 to commercialize liquid repellent treatments developed for military defense in the United Kingdom. The company currently holds 46 patents, most of which are related to electronics. According to the P2i Annual Corporate Review, 2010, three of the five world's largest hearing aid manufactures are currently using P2i's AridionTM liquid repellent coating, and approximately $50 \%$ of the globally manufactured hearing aids use (or will soon use) AridionTM.

P2i's Nano-coating technology works by applying a nanometerthin polymer over the entire surface of the product. ${ }^{4} \mathrm{P} 2 \mathrm{i}$ uses ionized gas to molecularly bind the thin layer to the surface of the product so the coating will not percolate away. The P2i Annual Corporate Review stated hearing aids using AridionTM technology have seen significant decreases in return rates, based on some three million units processed to date (published research could not be found to substantiate this claim). P2i also claims AridionTM substantially reduces in-warranty failure and repair cost, and lasts as long as the material it protects (2010).

\section{Sweat}

Valente $^{1}$ noted Buckley and Lewis (1960) reported a concentration of 0.5 to $0.85 \%$ of sodium chloride $(\mathrm{NaCl})$ can corrode mild steel. Valente suggests the impact of battery rust on hearing aid performance is likely minimal (at first) until corrosion builds up (over time) which leads to poor battery contact within the battery compartment, which eventually leads to intermittency or a "dead" hearing aid.

Of note, patients with recurrent moisture problems with their hearing aids and/or rust in their hearing aid battery door compartment may discontinue wearing hearing aids if the individual feels embarrassed that the rust reflects how well they care for their hearing aids or indicates their overall personal hygiene. ${ }^{2}$ Common chemicals found in sweat are $\mathrm{NaCl}$, potassium, urea, creatine, creatinine, lactate, and phosphate. Potassium levels were found to be higher in children's sweat when compared to adults. ${ }^{5}$ The chemical that has the greatest concentration is sodium chloride $(\mathrm{NaCl})$, approximately 1.8 grams/ liter. 
There are two types of sweat glands found in the human body; eccrine and apocrine. ${ }^{5}$ Sweat glands are distributed throughout the body and while both are present at birth, apocrine glands are believed "functional" at puberty. Environmental thermoregulation differs between children and adults. ${ }^{5}$ The ISO $3160 / 2$ is the international standard for the composition of "artificial sweat" and declares $\mathrm{NaCl}$ (in sweat) at 20 grams/liter (2007). During exercise the metabolic heat produced by the body may be 15 to 20 times higher than production at rest. ${ }^{5}$ Variation of the chemical makeup of sweat can be based on intensity and duration of exercise, sodium loss, sweat rate, gender, state of physical fitness, age, temperature, diet, type of clothing worn, humidity, acclimatization, hydration level, and diseases such as pancreatic cystic fibrosis. ${ }^{1}$ Due to the temperature sensitivity of ISO $3160 / 2$, artificial sweat was not a feasible variable for this study.

Further, sweat has a significant inter and intra subject variation. Children rely more on dry heat loss and less on evaporative cooling. ${ }^{5}$ Lower sweating rates of children are due to lower sweating rates per gland, as opposed to a lower number of sweat glands. For this reason it can be assumed moisture anomalies from perspiration are more common in adults than children. Two hormones which may be related to sweat gland function and or sweat composition differ between adults and children are testosterone and prolactin. ${ }^{5}$

Sweat rates can also fluctuate from 1 to 3 liters per hour with varying loss of $\mathrm{NaCl}$. A study conducted to analyze water and salt balance of well-trained swimmers in training, by Maughan, Dargavel, Hares and Shirreffs, ${ }^{6}$ concluded a mean sweat-volume loss of $548 \pm$ $243 \mathrm{ml}$ and a mean sweat rate of $0.31 \pm 0.1 \mathrm{~L} / \mathrm{hr}$. Mean sweat $\mathrm{Na}+$, $\mathrm{K}+$, and $\mathrm{Cl}$ - concentrations were $43 \pm 14,4 \pm 1$, and $31 \pm 9 \mathrm{mmol} / \mathrm{L}$, respectively. The maximal sweating rate in adults and children is unknown. ${ }^{5}$ Results in this study were also in agreement with previous aquatic studies.

\section{Chlorine (Table I)}

In 1774, Carl Scheele discovered the element chlorine. ${ }^{7}$ Chlorine is produced by the electrolysis of salt water and is used for sanitizing. When chlorine is added to water, the reaction is hypochlorous acid and hydrochloric acid. The quantity of each is dependent on water temperature and $\mathrm{pH} .{ }^{7}$ Forms of chlorine used commonly in commercial pools include: chlorine gas, sodium hypochlorite, calcium hypochlorite, lithium hypochlorite or chlorinated isocyanurates. ${ }^{8}$ According to the Association of Pool and Spa Professionals, the chart above represents suggested chemical standards for swimming pools. ${ }^{8}$

Table I Standard chlorine protocol measures

\begin{tabular}{ll} 
Free Chlorine, ppm & $2.0-4.0$ \\
$\mathrm{pH}$ & $7.2-7.8$ (ideal $7.4-7.6$ ) \\
Total alkalinity & $80-100$ \\
Total Dissolved Solids, ppm & Not to exceed I500 \\
Calcium hardness, ppm & $200-400$ \\
Cyanuric acid, ppm & $30-50$ \\
\hline
\end{tabular}

\section{Methodology}

Thirty hearing aids from 5 manufacturers were programmed to function full on gain. The 5 manufacturers were classified as A, B, C, D \& E. Technology level was not considered an important factor for this particular study, although most manufacturers provided top-ofthe-line hearing aids.

Of note, the majority of moisture studies conducted by hearing aid manufactures do not incorporate human hair into their experiments.
However, the objective of this research was to conduct a more accurate simulation of real world settings, and therefore human hair was considered a significant module to include in this study. The human hair was attached to the silicone display ears (Figure 1).

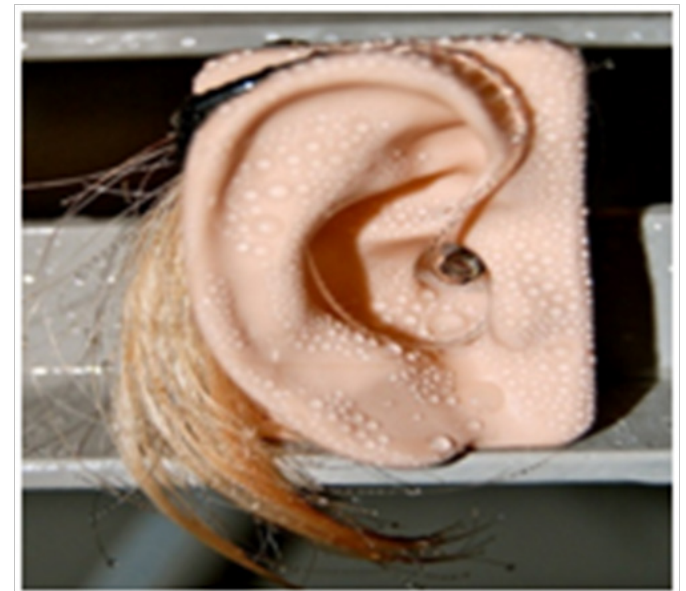

Figure I Silicone ear with human hair exposed to chlorinated water.

Starkey Research Laboratories found statistically significant results when using hair as opposed to not using hair. As sweat gathers around the hearing aid it may seep into the hearing aid case, which houses the battery and electrical components, leading to corrosion or causing moisture related hearing aid damage.

\section{WTP-60 Spray Chamber (Figure 2)}

The experiment evaluated Nano-coated hearing aids using a WTP60 spray chamber, which is an instrument designed for corrosive atmospheric tests, and in agreement with ASTM B117 standards. The WTP-60 is constructed with Polypropylene and PVC to function perfectly in acidic and alkaline environments without damage over time. The shelves that support the specimens within the chamber are Lucite (to eliminate the risk of corrosion). The chamber is designed to perform traditional salt spray (fog) and humidity tests following requirements and standards such as ISO, JIS, DIN, ASTM, IEC, GB, and SAE. ${ }^{9}$ The WTP-60 was programmed to mist the hearing aids on an intermittent timer throughout the 30minute, 180minute and 480minute exposure durations. All hearing aids were misted for 60 seconds followed by a 60 second static "rest" period.

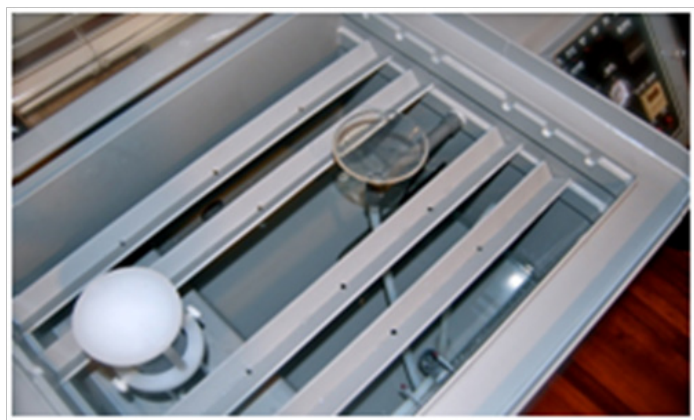

Figure 2 Arial view of the WTP-60 spray chamber.

\section{Batteries}

A zinc-air battery is a transducer, which converts one form of energy into another. It takes a small amount of oxygen to activate a zinc-air battery. Tiny air holes are located on the bottom (positive side) of the battery cannula. The major concern with hearing aid 
batteries is their susceptibility to environmental conditions. The tiny air holes and crevasses located on the battery may provide a prime location for moisture accumulation leading to corrosion and hearing aid malfunction. The effect of temperature can also degrade the performance of zinc-air batteries by reducing the ionic diffusion capability through the electrolyte. The optimum temperature for battery function is between $10-40^{\circ} \mathrm{C}$, but batteries still function at lower temperatures.

Four mechanisms affect the capacity of zinc-air cells during operation and storage; self-discharge of the zinc (corrosion), direct oxidation of the zinc anode, carbonation of the electrolyte, and electrolyte water gain or loss. Typically zinc-air cells have a shelf life of 5years yielding less than $2 \%$ capacity loss per year. It is noted that elevated temperatures will dramatically increase the rate of selfdischarge reaction. A cell containing 30\% concentration of potassium hydroxide will gain water at humidity above $60 \%$. Water gain dilutes the electrolyte and reduces conductivity. The catalyst layer of the cathode floods, reducing electrochemical activity, and causes the cell to fail.

Valente, et al., ${ }^{1}$ examined 320 zinc-air batteries from four manufacturers (Energizer, Power One, Ray-O-Vac, and Duracell) and incorporated four battery sizes $(10,312,13,675)$. The batteries were exposed to saltwater in a salt spray fog apparatus for durations of 2.5, 5.5, 24, 48, and 72 hours. Four experienced audiologists graded the presence of rust on a four-point scale. Initially rust was difficult for raters to observe, which was one of the points previously stated by BHI. Further analysis done by Valente, et al., ${ }^{1}$ using imaging (2000x magnification) and Auger Spectra analysis concluded Energizer and Power One had significantly less rust than Ray-O-Vac and Duracell. Of note, battery size was not considered a factor in the degree of rust. Based on these findings, the study used a total of 60 (sizes 312 and 13) Energizer batteries to allow for the best possible test results. The hearing aids used the same battery for 5 days and on day 6 a new battery was introduced.

Upon conclusion of the study all batteries were sent to a battery expert to rate the battery rust level based on a four-point scale and $\mathrm{X}$-Ray imaging.

\section{Listening Check \& Rating Rust Scale (Figure 3)}

Qualitative investigation included a listening check, which classified the hearing aid as either within normal limits (WNL), weak, intermittent or dead. Listening checks were taken 3times a day for 10 consecutive days on each hearing aid. Initial listening checks were taken before the hearing aid was exposed to either saltwater or chlorinated water, and again were repeated after being intermittently misted for 30 minutes, 180 minutes or 480 minutes with salt water or chlorinated water. Finally, listening checks were taken after the hearing aid concluded the $8 \mathrm{hr}$ session in the Dry and Store. A Dry and Store is an electronic appliance to store and care for hearing instruments over an 8hour period. There are three components which make up effective drying: heat, moving air, and a desiccant to absorb humidity.
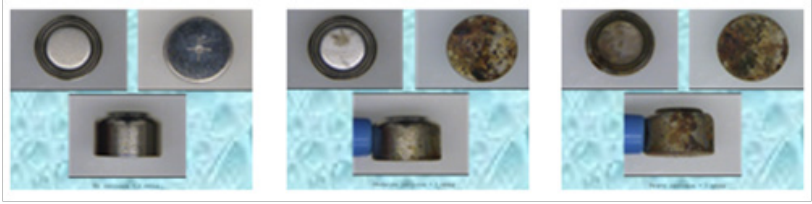

Figure 3 Rating Rust Scale example of 0,2 \& 3.
Further qualitative analysis asked an experienced battery expert to rate the battery rust level based on a four-point scale (0-3) as seen below. The cost of hearing aid repairs and the potential for patients to return hearing aids out of frustration due to moisture related malfunction necessitates an examination of the effects of moisture related environmental conditions on hearing aids. ${ }^{10}$

Rating Rust Scale:

$0=$ No rust observed via the naked eye or $10 \mathrm{x}$ magnification level; no corrosion.

$1=$ Rust observed only at the $10 \mathrm{x}$ magnification level; light corrosion.

$2=$ Rust noticeable via the naked eye; moderate corrosion.

$3=$ Rust easily observed via the naked eye; heavy corrosion.

*Rust rating scale model from study by Valente, et al., ${ }^{1}$

\section{Electro acoustic analysis (EAA) \& Weight}

Quantitative investigation incorporated electro acoustic analysis (EAA) to directly observe the impact of moisture using frequency specific distortion. This test measures harmonic distortion at the 2nd or 3rd harmonics with input levels varying from 60-90dB SPL. The total harmonic distortion (THD) is the sum of the 2nd and 3rd harmonic. The Total Harmonic Distortion for this experiment used an input level of $65 \mathrm{~dB}$ across frequencies $250-4000 \mathrm{~Hz}$. Max OSPL90, Average OSPL90, and average gain at $50 \mathrm{~dB}$ were observed. All hearing aids were tested in the Audio Scan, Verifit, VF-1, and RealEar Hearing Aid Analyzer. Specifically, the experiment recorded the ANSI-S3.22-2003 AGC. EAA measures were taken 3times a day for 10 consecutive days on each hearing aid See Figure 4.

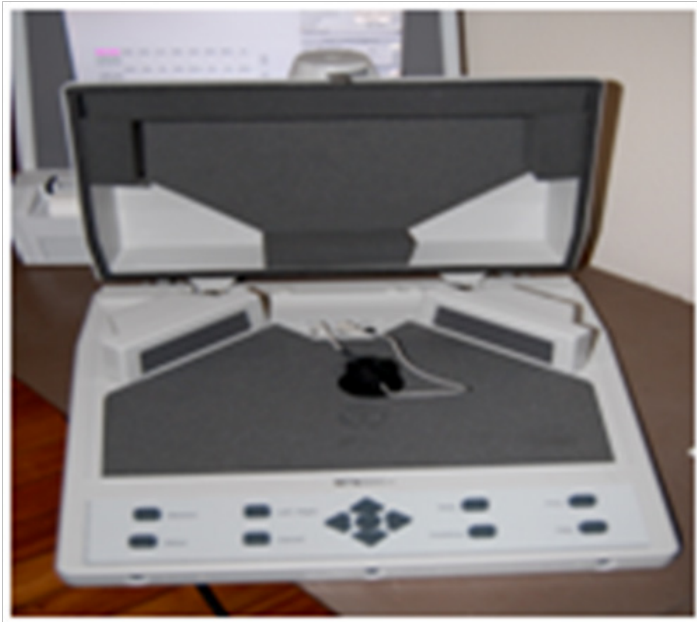

Figure 4 Audio scan Verifit seen above.

Initial EAA measures were taken before the hearing aid was exposed to (either) saltwater or chlorinated water. EAA measures were repeated after being intermittently misted for either 30minutes, 180 minutes or 480 minutes with salt water or chlorinated water. Finally, EAA measures were taken after the hearing aid concluded the $8 \mathrm{hr}$ session in the Dry and Store. Further quantitative analysis included hearing aid weight measured on a $0.0 \mathrm{~g}$ calibrated scale and $\mathrm{X}$-Ray battery cell imaging. Hearing aid weights were taken 3 times a day for 10 consecutive days on each hearing aid. Initial weight was taken before the hearing aid was exposed to either saltwater or chlorinated water. Hearing aid weights were repeated after being 
misted for 30 minutes, 180 minutes or 480 minutes with salt water or chlorinated water. Finally, hearing aid weight was taken after the hearing aid concluded the $8 \mathrm{hr}$ session in the Dry and Store.

\section{Options to Help Keep Hearing Aids dry}

Immediately following the specific exposure duration, the hearing aids were placed in an electronic Dry and Store for eight hours. When the hearing aids were not in the exposure duration or Dry and Store conditions, the hearing aids were individually placed in hard cases supplied by each manufacturer with the battery door open until the next test began or terminated. The Better Hearing Institute states hearing healthcare providers can offer dry packs, which absorb moisture, to decrease the probability of hearing aid failure related to moisture. The Hearing Aid Sweat BandTM, Dry and Store kits, hearing aid air blowers, and nano-coating technology are all tools and resources used for hearing aid maintenance related to moisture.

\section{Durations}

The environmental conditions evaluated were chosen based on close proximity to the Gulf Coast. The use of chlorinated water is based on the fact that some individuals engage in therapeutic activities in a pool such as a Hubbard tank or whirlpool, or athletic activities in a swimming pool.

\section{Results}

There was a significant two-way interaction between simulation condition and exposure duration, $F(2,384)=6.996, p<.00001$.

Distortion levels were higher in the saltwater condition than in the chlorinated condition for the 30 minute and 180 minute exposure durations. However, distortion levels were higher in the chlorinated condition than in the saltwater condition at $480 \mathrm{~min}$ see Figure 5. It was noted intermittent hearing aids had varying levels of distortion during intermittency. The time at which the measurement was taken for intermittent hearing aids significantly affected distortion levels, $F(5,1920)=22.787, p<.00001$. Regardless, spec sheets for most hearing aid manufacturers indicate distortion levels below 2 across frequencies. Distortion levels tended to be higher across days 6-10 than on days 1-5. The peak was observed for the post measurement across days 6-10 $(M=5.305$; Figure 6 .

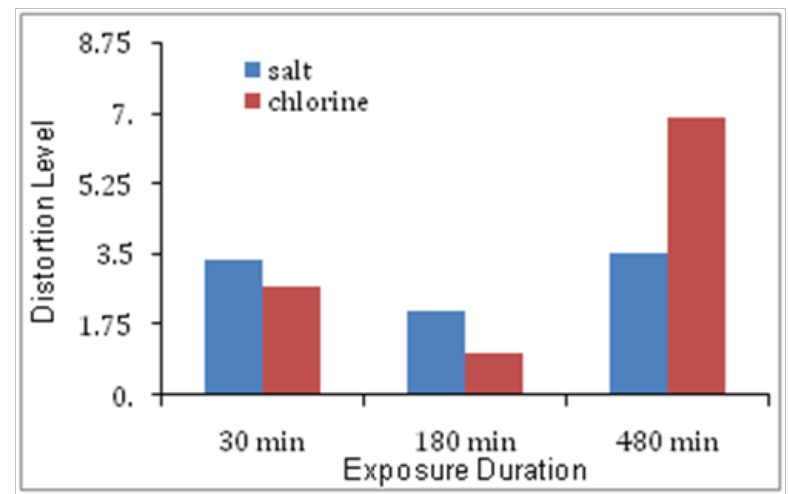

Figure 5 Mean distortion levels on days I-5 and days 6-10 as a function of time measurement (pre-exposure, exposure and post-exposure).

This rule out battery distortion involvement. If a new battery was replaced on day 6 and distortion levels were still the same or elevated one can conclude the battery is not a major implication of distortion level. There was a significant two-way interaction between simulation condition and time of measurement, $F(5,1920)=7.738, p<.00001$.
Distortion levels tended to be similar for both simulation conditions across the 6time points, with the exception of exposure 5 (average across days 1-5 during exposure). At this time, distortion levels were much higher in the chlorine condition $(M=4.444)$ than in the saltwater condition $(M=0.953$; Figure 7 .

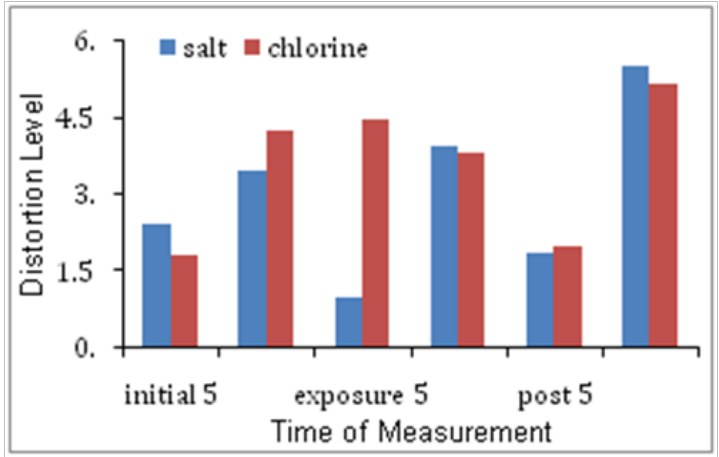

Figure 6 Cumulative distortion levels for saltwater and chlorinated water as a function of duration exposure ( 30 minutes, 180 minutes and 480 minutes).

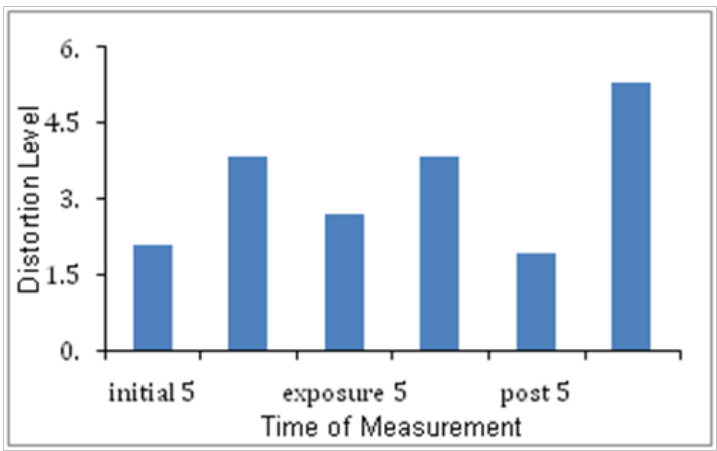

Figure 7 Mean distortion levels on days I-5 and days 6-10 as a function of simulation condition (saltwater and chlorinated water) and time measurement (pre-exposure, exposure and post-exposure).

There was a significant two-way interaction between exposure duration and time of measurement, $F(10,1920)=21.407, p<.00001$. Distortion levels tended to be highest for the $480 \mathrm{~min}$ exposure conditions, particularly over days 6-10 Figure 8 . It can be concluded that the longer the hearing aid is exposed to condition elements the more distortion will be identified.

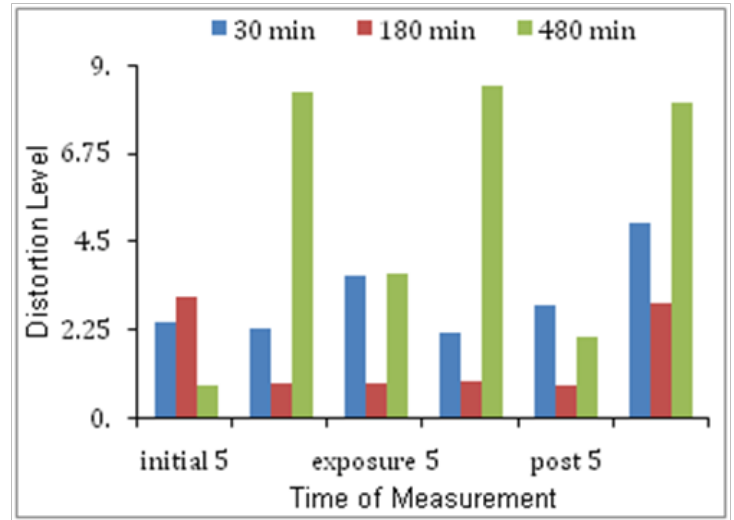

Figure 8 Mean distortion levels as a function of exposure duration (30minutes, 180 minutes, and 480 minutes) and time of measurement (preexposure, exposure and post exposure).

There was a significant three-way interaction between simulation condition, exposure duration, and time of measurement, $F(10,1920)$ 
$=9.134, p<.00001$. In general there was more variability in distortion levels in the chlorinated simulation condition across measurement times and exposure durations than in the saltwater condition. Distortion levels tended to be highest in the $480 \mathrm{~min}$ exposure duration across days6-10 for the chlorine condition. In the salt water condition, peaks in distortion were observed for all measurement times except days 1-5 during exposure, and across all three exposure durations see Figure $9 \& 10$.

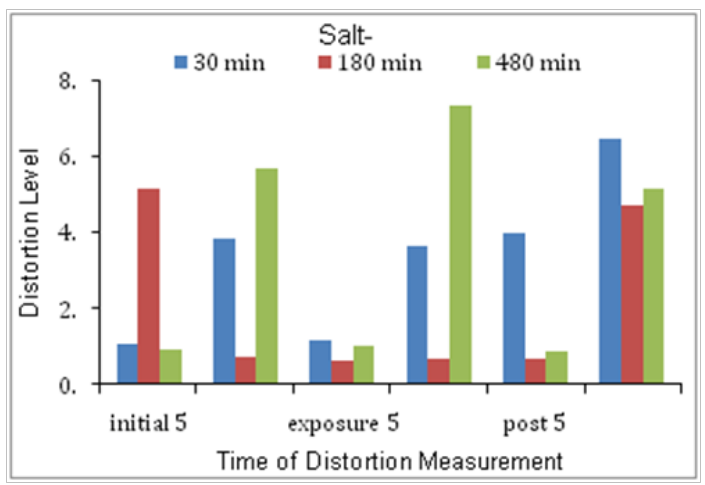

Figure 9 Mean saltwater distortion level as a function of exposure duration (30minutes, 180 minutes, and 480minutes) and time of measurement (preexposure, exposure and post exposure).

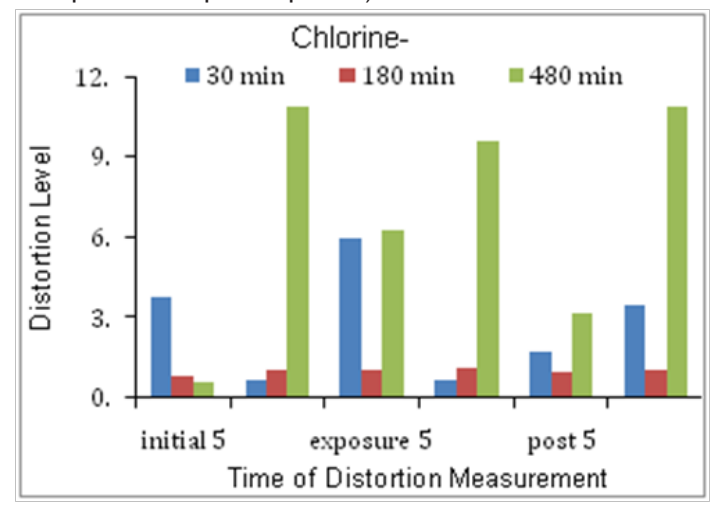

Figure 10 Mean chlorinated water distortion level as a function of exposure duration (30minutes, 180 minutes, and 480 minutes) and time of measurement (pre-exposure, exposure and post exposure).

\section{Discussion}

Relatively few published studies have examined the impact of moisture on hearing aids and or examined the effects of different types of moisture (e.g. sweat, saltwater, or chlorinated water) on hearing aids. Moisture and electronics often leads to corrosion and failure of the electronic device. Common problems for hearing aids include: electrical shortages, corrosion, condensation, and are often the leading cause for hearing aid repair. ${ }^{11}$ Water vapor arising from the ear canal condenses in the connecting tube, and once the vapors reach a region outside the canal (which in most cases may be a slightly cooler temperature) condensation appears. The diameter of BTE hearing aid tubing is small, and condensation or moisture is easily transferred from the tip of the hearing aid to the instrument itself.

For example, take a simple physics experiment involving three bottomless cylinders with large, medium and small diameters placed in a liquid bath. The tube with the smallest diameter will have the highest liquid level over a given period. This concept can be related to a vacuum or the diameter of a pressure washer. The accumulation of enough condensation can shut down amplification. ${ }^{2}$ When the hearing aid is placed deep inside the canal, condensation is less likely to affect the internal and external components of the hearing aid.

Perspiration is another form of moisture concerning hearing aids, and beads in the hair along the top of the hearing aid. The moisture that forms in the hair along the top of the hearing aid is more likely to permeate the hearing aid circuitry, and consequently has more deleterious effects. ${ }^{2}$ Individuals who reside or visit regions of the world with higher humidity levels may be at higher risk for moisture related problems with their hearing aids due to increased level of sweat production. Hearing aid consumers who make up America's workforce requiring environmental outside labor may benefit from new information concerning their hearing aids or persuade new hearing aid candidates to purchase hearing aids. BHI reported sweat is the leading cause of moisture related anomalies found in hearing aids. ${ }^{2}$ Reports on the incidence of moisture related problems as the primary cause of hearing aid malfunction varies dramatically. According to Valente, et al., ${ }^{1}$ of the eight major hearing aid manufacturers contacted, moisture related anomalies ranged from $1 \%-100 \%$ of all repairs. BTEs were determined to be more susceptible to moisture as compared to ITE hearing aids, which is in agreement to BHI's moisture theory previously stated. ${ }^{12-16}$

\section{Conclusion}

The results from this study answered the following questions.

a. Moisture was significantly difficult to observe without electro acoustical analysis. A combination of quantitative and qualitative data should be used. A listening check and the rating rust scale were not enough to identify moisture in most cases. However, EAA may not always represent consistent distortion levels for intermittent hearing aids.

b. Significant differences were present in the relationship of exposure duration. The longer the hearing aid was exposed to the simulated condition (saltwater and chlorinated water) the greater the impact on the distortion levels, overall gain and degraded listening checks.

c. Significant differences were not present between saltwater and chlorinated water except for the saltwater exposure condition during days 1-5 as seen in figure 8. Intermittency may have played a role, but regardless the average distortion across most hearing aids was elevated.

Significant differences were not present in the relationship of hearing aid function on days 1-5 and 6-10. These findings further support that the battery is not the main cause of hearing aid distortion.

\section{Acknowledgements}

I wish to thank the following people for helping fund, collaborate and edit this project: Annette Mazevski, Au.D., Oticon Manager; Brian Taylor, Au.D., Unitron Director, Practice Development \& Clinical Affairs; Thomas Powers, Ph.D., Siemens, Vice President, Compliance Officer; Brian Sprague, Energizer, Sr. Technologist; Michel Levy, Audio Electronics; Edythe Kirk, Ph.D., Lamar University, Psychology; Shannon Frugia, Au.D., South East Texas ENT, Audiologist; Jason Johnson, M.A., Unitron, Account Executive; Paul Giffin, Unitron, Sales; Michael Valente, Ph.D., Washington University School of Medicine; Kerwin Nealer, Auto Technology Company; Lamar University Speech and Hearing Clinic; Denise McLeod, Au.D., Oticon, Account Manager; Douglas Beck, Au.D., Director of Academic Science, Oticon Inc.; Davey Shaw, Weice 
Testing Instrument Co., Ltd., Oversea Sales Manager; David Gordon, M.S., Oticon, Account Manager-Pediatrics; Crystal Wiggins, Au.D., Texas Center for Hearing, Audiologist; Donald Schum, Ph.D., Oticon, Vice President, Audiology \& Professional Relations; Laurel Christensen, Ph.D., VP Research and Development; Michael Thomas, Siemens, Senior Account Representative; Scott Witt, Phonak and Nathan Franz, Research Engineering \& Operations Manager. Douglas L. Beck, Au.D., Director of Professional relations, Oticon Inc.

\section{Conflicts of interest}

Author declares there are no conflicts of interest.

\section{Funding}

None.

\section{References}

1. Valente M, Cadieux JH, Flowers L, et al. Differences in rust in hearing aid batteries across four manufacturers, four battery sizes, and five durations of exposure. J Am Acad Audiol. 2007;18(10):846-862.

2. Schow RL, Brockett J. Your Guide to Care and Maintenance of Hearing Aids. Better Hearing Institute. 2011

3. Strom K. Hearing Aid Sales Holding Steady. The Hearing Review. 2011.

4. P2i.. The world leader in liquid repellent nano-coating technology. 2010:1-68.
5. Falk B. Effects of thermal stress during rest and exercise in the pediatric population. Sports Med. 1998;25(4):221-240.

6. Maughan RJ, Dargavel LA, Hares R, et al. Water and Salt Balance of Well-Trained Swimmers in Training. Int J Sport Nutr Exerc Metab. 2009;19(6):598-606.

7. Chlorine Chemistry. Pool center. 2011

8. Pool Treatment 101: Introduction to Chlorine Sanitizing. American Chemical Council. 2011

9. Standard Practice for Operating Salt Spray (Fog) Apparatus. ASTM International. 2012;3(2):1-11.

10. Bongiovanni R, Jordan JA. Fitting Hearing Aids and Getting the Patient to Keep Them Are Two Different Things. Phonak University, Chicago, Phonak, USA. 2011. P. 27.

11. Chalupper J, Flores A. Beneath the Surface: Understanding the "Water Resistant" and "Waterproof". The Hearing Review. 2011;18(11):56-60.

12. Coulson SR, Woodward IS, Badyal JS, et al. Ultralow Surface Energy Plasma Polymer Films. Chem Mater. 2011;12(7):2031-2038.

13. Quick Statistics about Hearing. National Institute on Deafness and Other Communication Disorders. 2010.

14. Oticon Pediatric Portfolio Safari and More. Oticon. 2011;1-39.

15. Siemens Aquaris. Siemens. 2011

16. Product Categories. Test Equipment Corporation. 2011 